\title{
Enhanced Photogeneration of Polaron Pairs in Neat Semicrystalline Donor-Acceptor Copolymer Films via Direct Excitation of Interchain Aggregates
}

\author{
Daniele Di Nuzzo, ${ }^{\dagger \dagger}$ Daniele Viola, ${ }^{\ddagger}$ Florian S. U. Fischer, ${ }^{\S}$ Giulio Cerullo, ${ }^{\ddagger}$ Sabine Ludwigs, ${ }^{*}, \S$ \\ and Enrico Da Como ${ }^{\dagger}$
}

${ }^{\dagger}$ Department of Physics, University of Bath, BA2 7AY Bath, United Kingdom

${ }^{\ddagger}$ Dipartimento di Fisica, IFN-CNR, Politecnico di Milano, 20133 Milano, Italy

${ }^{\S}$ IPOC-Functional Polymers, University of Stuttgart, 70569 Stuttgart, Germany

\section{Supporting Information}

ABSTRACT: We investigate the photogeneration of polaron pairs (PPs) in neat films of the semicrystalline donor-acceptor semiconducting copolymer PCPDTBT. Carefully selecting the solution-processing procedures, we obtain films with different amounts of crystallinity and interchain aggregation. We compare the photogeneration of PPs between the films by monitoring their photoinduced absorption in ultrafast pump-probe experiments, selectively exciting nonaggregated or aggregated polymer chains. The direct photoexcitation of interchain $\pi$-aggregates results in prompt $(<100 \mathrm{fs})$ charge generation. Compared to the case where nonaggregated chains are excited, we find an 8-fold increase in the prompt PP to singlet-exciton ratio. We also show that highly crystalline lamellar nanostructures not containing $\pi$-stacked or any light-absorbing aggregates do not improve the efficiency of PP photogeneration. Our results show that light absorption from interchain aggregates is highly beneficial for charge photogeneration in semiconducting polymers and should be taken into account when optimizing film morphologies for photovoltaic devices.

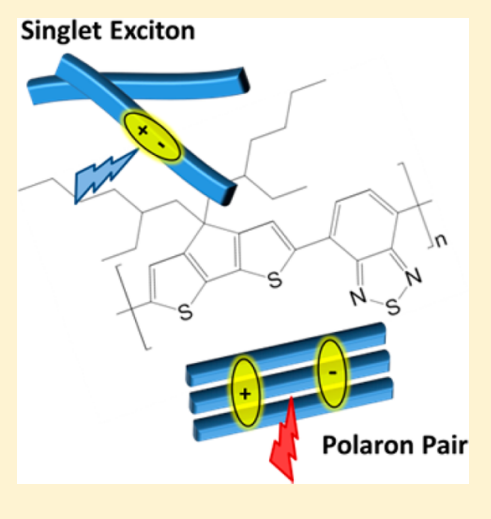

$I^{n}$ n sharp contrast with inorganic semiconductors such as silicon, the photogeneration of free charges in semiconducting conjugated polymers is inefficient, with the absorption of light resulting instead in the creation of tightly bound electron-hole pairs (singlet excitons, SEs). This is due to the poor Coulomb screening typical of conjugated polymers. To enable their employment in optoelectronic devices based on the photogeneration and extraction of charge carriers, such as photovoltaic solar cells, conjugated polymers are normally mixed with a second electron-accepting material to form a bulk heterojunction; ${ }^{1}$ the donor-acceptor junction provides the necessary driving force for the dissociation of photogenerated SEs into spatially separated electron-hole pairs.

Yet, photogeneration of charged states has been observed in neat films of conjugated polymers. ${ }^{2-11}$ These charged states can be generically defined as polaron pairs (PPs); in a PP, the electron and hole are more separated in space compared to an exciton. Free charges can be considered as a special case of PPs, for which the separation is such that the Coulomb attraction between the electron and hole is negligible. The photogeneration yield of PPs in neat polymers is normally small $(<20 \%)$, with most of the absorbed photons resulting in SEs. ${ }^{7}$ Furthermore, photogenerated PPs recombine too quickly $(<0.5 \mathrm{~ns})^{3,4,7,11}$ to be extractable from the electrodes and thus contribute to the photocurrent in a photovoltaic solar cell.

Nevertheless, the mechanism of PP photogeneration in neat polymers could be relevant for the efficiency of solar cell devices.
Indeed, the bulk heterojunctions used in polymer solar cells can contain pure or relatively pure polymer domains with sizes of a few tens of nanometers; ${ }^{12-15}$ therefore, above the typical SE diffusion length $(<10 \mathrm{~nm}) ;{ }^{16,17}$ one could argue that PPs directly photogenerated inside of large polymer domains can travel to the heterojunction and be further separated into free charges, therefore contributing to the overall photogenerated current. Furthermore, the separation of PPs at the heterojunction might be easier than that for the interfacial charge transfer states (CTSs), which are created upon SE splitting. This would be due to a larger electron-hole distance and hence a lower Coulomb attraction.

Ultrafast optical spectroscopy studies of the process of charge generation in bulk heterojunctions have shown examples where a non-negligible amount of charges is generated promptly, that is, in the first $100 \mathrm{fs}$ after photoexcitation. ${ }^{18,19}$ It is possible that some of these charges are generated from PP precursors in one of the two materials of the bulk heterojunction. However, the interpretation of optical spectroscopy data in a heterojunction is complicated by other competing processes; indeed, prompt charge generation might be observed from SEs generated close to the donor-acceptor interface or by direct excitation of interfacial high-energy CTSs. ${ }^{20,21}$ Overall, the similarity in the excited-state

Received: February 1, 2015

Accepted: March 16, 2015

Published: March 16, 2015 
absortpion spectra of PPs, CTSs, and separated free charges makes the interpretation of such experiments arduos.

Recently, evidence of the influence of interchain interactions and intermolecular order on the process of photogeneration of PPs in neat polymer films has emerged. ${ }^{22-26}$ In polymers such as poly(3-hexylthiophene) ( $\mathrm{P} 3 \mathrm{HT})$, the crystal structure and the mesoscopic morphology are well established; ${ }^{27}$ in addition to nonaggregated, amorphous regions with coiled polymer chains having only weak interchain coupling and low conjugation length, aggregates are present based on long-range $\pi$-stacking and strong electronic coupling between the chains. It has been suggested that the energy offset between the molecularly disordered and ordered phases described above might serve as a driving force for charge separation. ${ }^{23,24,26}$ Furthermore, evidence that direct PP photogeneration benefits from interchain interactions has been shown in homopolymers such as $\mathrm{P} 3 \mathrm{HT}$ and PPV derivatives. ${ }^{22}$

Here, we address the photogeneration of PPs in neat polymer films and its dependence on film nanomorphology, focusing our attention on the important class of conjugated donor-acceptor copolymers. The choice of studying neat films allows us to avoid the above-mentioned complications of bulk heterojunctions and to concentrate on the variety of photophysical processes taking place in polymers in the solid state. We use ultrafast optical spectroscopy to study neat films of the semicrystalline, smallband-gap donor-acceptor copolymer PCPDTBT (Figure 1), comparing amorphous films with semicrystalline films with and without long-range $\pi$-stacking in the crystalline domains. ${ }^{28,29}$

The films studied in this Letter were prepared by using different processing and postannealing conditions. We showed in a)

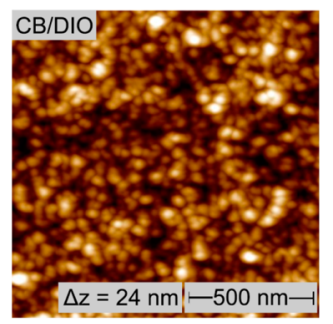

b)

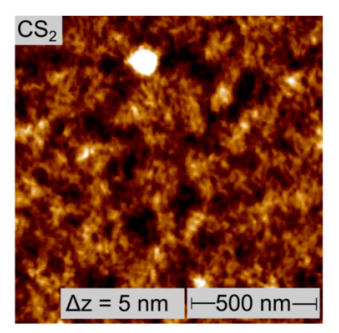

c)

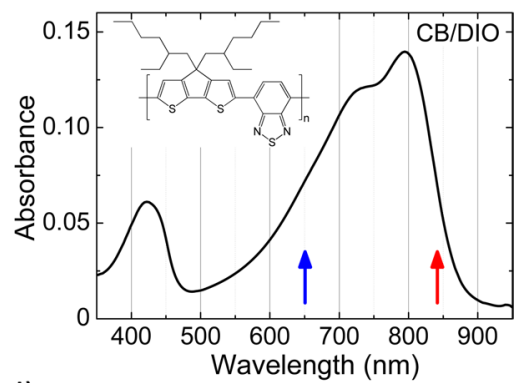

d)

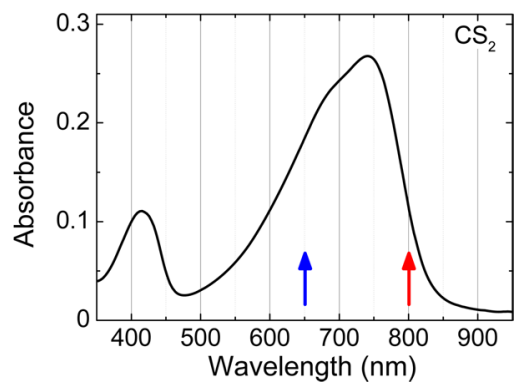

Figure 1. (a) AFM height image and (c) absorption spectrum of a neat PCPDTBT thin film spin-coated from CB with 2 wt \% DIO as an additive ("sample-CB/DIO"). The inset in (c) shows the chemical structure of PCPDTBT. (b) AFM height image and (d) absorption spectrum of a thin films of neat PCPDTBT spin-coated from $\mathrm{CS}_{2}$ ("sample- $\mathrm{CS}_{2}$ "). The absorption spectra were measured at room temperature $\left(25{ }^{\circ} \mathrm{C}\right)$. In (a) and (b), $\Delta z$ corresponds to the color scale. In (c) and (d), the blue and red arrows indicate the different excitation wavelengths used in the pump-probe experiments. recent publications that the film morphology and crystal structure of neat PCPDTBT films can be tuned by the choice of the processing solvent and solvent vapor annealing treatment. ${ }^{28,29}$ The use of low-boiling-point solvents, such as carbon disulfide $\left(\mathrm{CS}_{2}\right)$, leads to rather amorphous films. On the other hand, semicrystalline films of two different crystal structures could be generated by the following two procedures: (i) following the established literature protocol for solar cells, which is based on spin coating from a chlorobenzene (CB) solution with 1,8-diiodooctane (DIO) as a solvent additive, thin films with long-range $\pi$-stacked aggregates could be prepared; (ii) swelling and recrystallization of precast spin-coated films by exposure to a $\mathrm{CS}_{2}$ solvent vapor atmosphere resulted in a new crystal structure giving rise to highly ordered semicrystalline lamellar nanostructures. TEM/ED and GIWAXS data of this new crystal structure gave no evidence of long-range $\pi$-stacking but rather suggested a dimer-like structure. ${ }^{29}$

In the ultrafast pump-probe experiments, we use different excitation wavelengths, selectively resonant with the groundstate absorption of aggregated or nonaggregated chains. We observe prompt $(<100 \mathrm{fs})$ photoinduced absorption (PIA) from PPs and from the lowest-energy SE state $\left(S_{1}\right)$, and we monitor its evolution in time. By using a global analysis of the transient absorption spectra, ${ }^{30}$ we disentangle the contributions from the different photogenerated species, showing that direct photoexcitation of $\pi$-aggregates results in an 8 -fold increase in the prompt PPs to SEs ratio compared to the case where nonaggregated chains are excited. On the other hand, the presence alone of highly ordered semicrystalline lamellar domains but lacking ground-state absorption from intermolecular aggregates does not result in an enhanced PPs to SEs ratio.

Figure 1 shows atomic force microscope (AFM) height images of two samples, a film of PCPDTBT spin-coated from a CB solution containing $2 \mathrm{wt} \%$ DIO (hereafter called sample-CB/ DIO, Figure 1a) and a film of the same polymer spin-coated from a $3 \mathrm{mg} / \mathrm{mL} \mathrm{CS} \mathrm{C}_{2}$ solution (hereafter called sample- $\mathrm{CS}_{2}$, Figure 1b).

The surface of sample-CB/DIO shows circular structures on the order of $40 \mathrm{~nm}$, while sample- $\mathrm{CS}_{2}$ presents a rather featureless surface. Note that the surface roughness of sample$\mathrm{CS}_{2}$ is lower than the one of sample-CB/DIO, as can also be seen by comparing the $\Delta z$ scales in Figure $1 \mathrm{~b}$ and a, respectively.

To gain more information on the interchain interactions in the different neat polymer films, we measured their optical absorption in the UV-vis-NIR part of the spectrum. The results are shown in Figure 1c,d. The spectra were recorded with the samples held at room temperature $\left(25^{\circ} \mathrm{C}\right)$. Sample-CB/DIO presents two absorption bands in the $600-850 \mathrm{~nm}$ region, one peaked at $\sim 800 \mathrm{~nm}$ and another peaked at $\sim 750 \mathrm{~nm}$ (Figure 1c). We assign the $800 \mathrm{~nm}$ band to absorption from $\pi$-aggregates, this attribution being corroborated by previous studies on the PCPDTBT system, as we are going to discuss. Previously, Peet et al. have assigned the $800 \mathrm{~nm}$ absorption band to $\pi$-aggregates in a study of aggregate formation in films of PCPDTBT cast from different solvents, including CB/DIO mixtures. ${ }^{31}$ The nanomorphology of thin films obtained by spin coating PCPDTBT from $\mathrm{CB} / \mathrm{DIO}$ solutions has been extensively addressed by several other research groups, all confirming the formation of $\pi$ stacked interchain aggregates. ${ }^{29,32-35}$ In particular, GIWAXS studies on such films showed that PCPDTBT chains form an edge-on morphology, similar to $\mathrm{P} 3 \mathrm{HT}$, comprising long-range $\pi$ stacking with an interchain stacking distance of $3.8 \AA^{32,35}$ Importantly, the combination of GIWAXS and optical 
a)

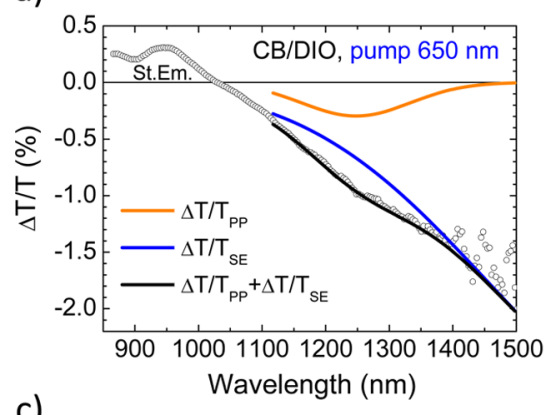

c)

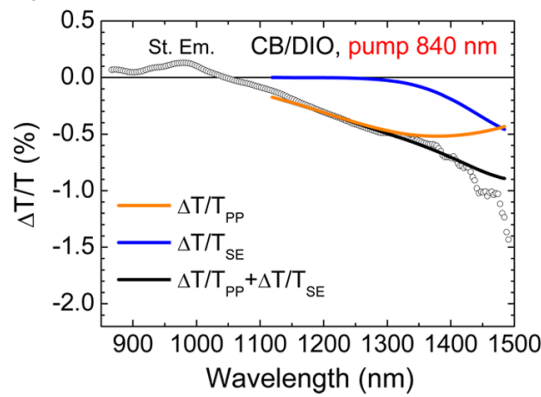

b)

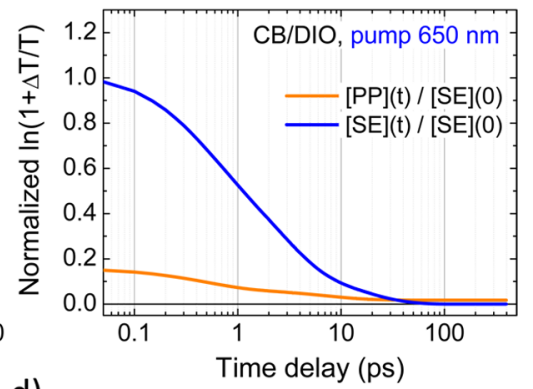

d)

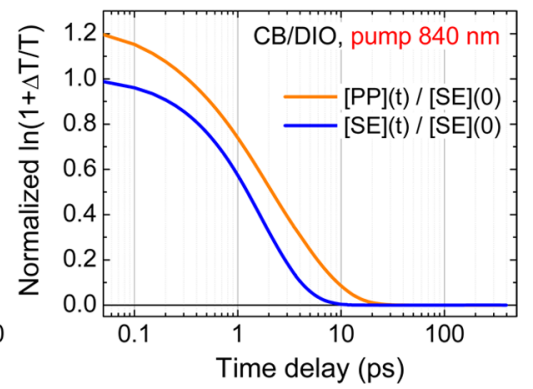

Figure 2. (a) Transient $\Delta T / T$ spectrum of sample-CB/DIO recorded $300 \mathrm{fs}$ after excitation with a pulsed pump at $650 \mathrm{~nm}$. The experimental spectrum (circles) is fitted with the sum (black line) of $\Delta T / T_{\mathrm{PP}}$ (orange line) and $\Delta T / T_{\mathrm{SE}}$ (blue line) (see eqs 2 and 3 ). The decay in time of $[\mathrm{PP}](t)$ and [SE] $(t)$ (see eqs 4 and 5) after the excitation is shown in (b), normalized to [SE](0). Panels (c) and (d) show the results on the same sample using a pump at 840 nm. In panels (a) and (c), "St. Em." indicates the signal from stimulated emission.

absorption experiments has confirmed the assignment of the strong band at $800 \mathrm{~nm}$ to absorption from $\pi$-aggregates. ${ }^{32,35}$

To further support the assignment to $\pi$-aggregates of the 800 $\mathrm{nm}$ band in sample-CB/DIO, we measure the spectra at different temperatures, from 25 to $100{ }^{\circ} \mathrm{C}$ (Supporting Information, Figure S1). By raising the temperature, the $800 \mathrm{~nm}$ band progressively disappears, until only the $750 \mathrm{~nm}$ band is left at 100 ${ }^{\circ} \mathrm{C}$. The band at $750 \mathrm{~nm}$, which is not influenced by the temperature of the sample, is attributed to absorption from nonaggregated polymer chains.

In sample- $\mathrm{CS}_{2}$, only the absorption from nonaggregated chains is present, peaked at $750 \mathrm{~nm}$ (Figure 1d). Confirming the assignment of this band to nonaggregated chains, the absorption spectrum of sample- $\mathrm{CS}_{2}$ coincides with that of PCPDTBT dissolved in $\mathrm{CS}_{2}$ (Figure S2, Supporting Information) and shows almost no change during temperature annealing. In summary, we interpret our optical absorption data in terms of interchain aggregation, based on previous studies that have characterized the subnanometer morphology in this type of films. ${ }^{28,29,31-35}$ Specifically, from the absorption data, we infer that sample-CB/ DIO contains a considerable amount of $\pi$-aggregates, while on the contrary, sample- $\mathrm{CS}_{2}$ does not contain a detectable amount of them. Furthermore, from our AFM images of Figure 1a,b we extract information on larger length scales and conclude that no long-range superstructures $(>40 \mathrm{~nm})$ are formed in either of the films.

To investigate the mechanism of charge photogeneration in the presence of interchain aggregates, we performed ultrafast pump-probe experiments on the neat PCPDTBT films; the evolution in time of the photoinduced excitations was studied comparing the effect of exciting the nonaggregated or the aggregated chains (pump wavelengths at 650 and $800-840 \mathrm{~nm}$, respectively). Figure $2 \mathrm{a}$ shows the differential transmission $(\Delta T /$ $T$ ) spectrum of sample-CB/DIO, probed at $300 \mathrm{fs}$ delay, upon selective excitation of the nonaggregated chains at $650 \mathrm{~nm}$. In the
$850-1050 \mathrm{~nm}$ wavelength range, we observe a positive $\Delta T / T$ signal, assigned to stimulated emission from SEs. Between 1050 and $1500 \mathrm{~nm}$, we observe a broad negative $\Delta T / T$ band, assigned to PIA from excited states. On the basis of previous reports from us and other groups, ${ }^{7,11,36-38}$ we attribute the shoulder at around $1250 \mathrm{~nm}$ to PIA from PPs and the signal at longer wavelengths to the tail of the $S_{1} \rightarrow S_{n}$ PIA from SEs. The assignment of the 1250 $\mathrm{nm}$ band to PP is further confirmed by spectroelectrochemistry experiments (Supporting Information, Figure S3) on oxidized PCPDTBT. To disentangle the contributions to the PIA from PPs and SEs, we performed a global analysis ${ }^{30}$ of the spectra and fitted the photoinduced signal with two Gaussian-shaped bands, one peaked at $1248 \mathrm{~nm}$ and the other at $1735 \mathrm{~nm}$ (Tables S1 and S2 in the Supporting Information). The evolution of the PIA spectra after photoexcitation was fitted to a function of the form

$$
\frac{\Delta T}{T}(\lambda, t)=\frac{\Delta T}{T_{\mathrm{PP}}}(\lambda, t)+\frac{\Delta T}{T_{\mathrm{SE}}}(\lambda, t)
$$

where

$$
\frac{\Delta T}{T_{\mathrm{PP}}}(\lambda, t)=f(\lambda) \cdot \sum_{i}^{3} A_{i} \cdot \mathrm{e}^{-t / \tau_{\text {ia }}}
$$

and

$$
\frac{\Delta T}{T_{\mathrm{SE}}}(\lambda, t)=g(\lambda) \cdot \sum_{i}^{3} B_{i} \cdot \mathrm{e}^{-t / \tau_{\mathrm{ib}}}
$$

In eqs 2 and $3, f(\lambda)$ and $g(\lambda)$ are Gaussian functions of the form $\mathrm{e}^{-(1 / 2)\left[\left(\lambda-\lambda_{0}\right) / \sigma\right]^{2}}$, modeling the PIA spectra of PP and SE, respectively. $\tau_{i \mathrm{a}}$ and $\tau_{i \mathrm{~b}}$ are the lifetimes of $\mathrm{PP}$ and $\mathrm{SE}$, respectively; in both cases, we opted for a triexponential decay function. The fitting functions in eqs 2 and 3 were derived from a kinetic description of the photoexcited system in which PPs and SEs are both directly populated from the ground state and their 
a)

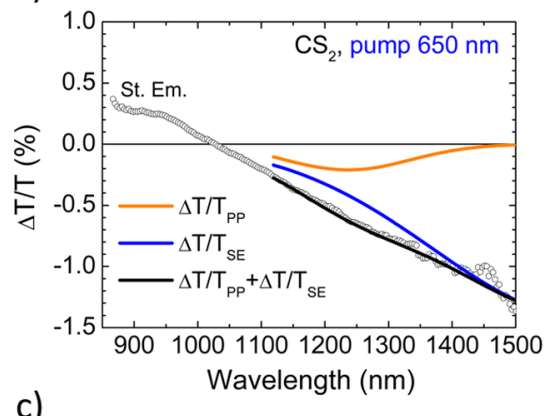

c)

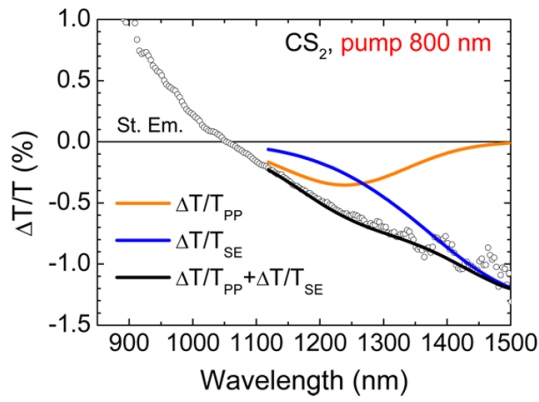

b)

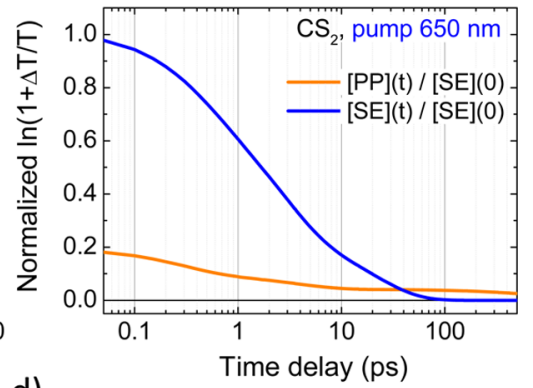

d)

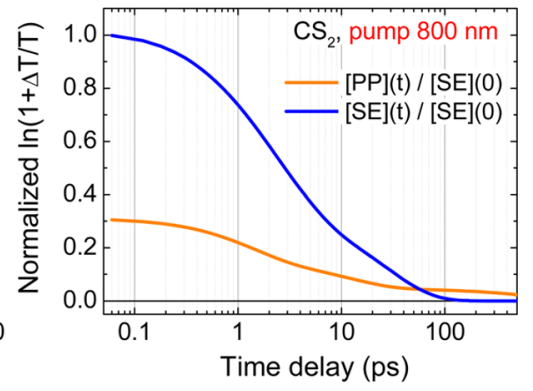

Figure 3. (a) Transient $\Delta T / T$ spectrum of sample- $\mathrm{CS}_{2}$ recorded $300 \mathrm{fs}$ after excitation with a pulsed pump at $650 \mathrm{~nm}$. The experimental spectrum (circles) is fitted with the sum (black line) of $\Delta T / T_{\mathrm{PP}}$ (orange line) and $\Delta T / T_{\mathrm{SE}}$ (blue line) (see eqs 2 and 3 ). The decay in time of $[\mathrm{PP}](t)$ and $[\mathrm{SE}](t)$ (see eqs 4 and 5) after the excitation is shown in (b), normalized to $[\mathrm{SE}](0)$. Panels (c) and (d) show the results on the same sample using a pump at 800 $\mathrm{nm}$. In panels (a) and (c), "St. Em." indicates the signal from stimulated emission.

evolutions in time are independent from each other. Tables $\mathrm{S} 1$ and S2 (Supporting Information) summarize the values of the best fit for the parameters used in eqs 2 and 3, respectively. In Figure $2 \mathrm{a}$, the best fits of eqs $1-3$ to the experimental data at 300 fs delay are shown. We chose a triexponential function in an attempt to describe the recombination of PPs and SEs in the presence of a distribution of decay rates, which is expected to exist in the disorder-broadened energy landscape of semiconducting polymers. We find that the triexponential is a useful description in that it allows us to isolate a long-lived component ( $>400 \mathrm{ps}$ ) in the decay of PPs observed in some of our measurements, which we discuss later. Alternative descriptions of the decay of photogenerated species in the presence of energetic disorder might be used, such as power-law functions. ${ }^{39,40}$ However, the discrimination between triexponential and power-law decays would require signal intensities on a much broader range ( $\geq 3$ orders of magnitude) than the one that we access in our work. Considering these reasons, we decided to use triexponential decay functions for the analysis of our data.

Figure $2 \mathrm{~b}$ shows the evolution in time of $[\mathrm{PP}](t) /[\mathrm{SE}](0)$ and $[\mathrm{SE}](t) /[\mathrm{SE}](0) .[\mathrm{PP}](t)$ and $[\mathrm{SE}](\mathrm{t})$ are defined as follows

$$
\begin{aligned}
& {[\mathrm{PP}](t) \equiv N_{\mathrm{PP}}(t) \cdot \phi_{\mathrm{PP}} \cdot d=\ln \left(1+\frac{\Delta T}{T_{\mathrm{PP}}}(t)\right)} \\
& =\ln \left(1+\sum_{i}^{3} A_{i} \cdot \mathrm{e}^{-t / \tau_{\mathrm{ia}}}\right)
\end{aligned}
$$

and

$$
\begin{aligned}
& {[\mathrm{SE}](t) \equiv N_{\mathrm{SE}}(t) \cdot \phi_{\mathrm{SE}} \cdot d=\ln \left(1+\frac{\Delta T}{T_{\mathrm{SE}}}(t)\right)} \\
& =\ln \left(1+\sum_{i}^{3} B_{i} \cdot \mathrm{e}^{-t / \tau_{i \mathrm{~b}}}\right)
\end{aligned}
$$

where $N_{\mathrm{PP}}(t)$ and $N_{\mathrm{SE}}(t)$ are the density of PPs and SEs, respectively, while $\phi_{\mathrm{PP}}$ and $\phi_{\mathrm{SE}}$ are their excited-state absorption cross sections and $d$ is the thickness of the film. Therefore, $[\mathrm{PP}](t) /[\mathrm{SE}](0)$ is the ratio of the density of PPs and SEs, multiplied by the ratio of their cross sections

$$
\frac{[\mathrm{PP}](t)}{[\mathrm{SE}](0)} \equiv \frac{N_{\mathrm{PP}}(t)}{N_{\mathrm{SE}}(0)} \cdot \frac{\phi_{\mathrm{PP}}}{\phi_{\mathrm{SE}}}
$$

As can be seen in Figure $2 \mathrm{~b}$, at time zero, $[\mathrm{PP}](t)$ is $15 \%$ of $[\mathrm{SE}](t)$. The triexponential decay for $[\mathrm{PP}](t)$ is characterized by the lifetimes of $\sim 500 \mathrm{fs}$ and $\sim 7 \mathrm{ps}$ and a long-lived tail, giving a residual PIA signal at $400 \mathrm{ps}$ (Table S1, Supporting Information). $[\mathrm{SE}](t)$ also presents a short lifetime on the order of $500 \mathrm{fs}$, while the other two decay lifetimes are shorter compared to $[\mathrm{PP}](t)$, with 2.5 and $\sim 16$ ps (Table S2, Supporting Information). This rather short lifetime of the SE is in agreement with previous reports on PCPDTBT ${ }^{11,36}$ and is characteristic of low-band-gap donor-acceptor polymers. ${ }^{41}$

Figure $2 \mathrm{c}$ shows the $\Delta T / T$ spectrum measured on the same film at $300 \mathrm{fs}$ delay, this time selectively exciting the $\pi$-aggregates by tuning the pump pulse to $840 \mathrm{~nm}$. Note that the intensities of all the $\Delta T / T$ spectra presented in the paper were scaled to take into account the variations in absorbance of the different samples at each pump wavelength. This was done by dividing the values of $\Delta T / T$ by the ratio between the number of absorbed photons at the pump wavelength and the absorbed photons at $650 \mathrm{~nm}$ in sample-CB/DIO. With the pump at $840 \mathrm{~nm}$, the PIA band of PP is red-shifted compared to the previous experiment, passing from 1248 to $1379 \mathrm{~nm}$ (Table S1, Supporting Information). The band is also broader and more prominent than that in the experiment with pump at $650 \mathrm{~nm}$. Figure $2 \mathrm{~d}$ shows $[\mathrm{PP}](t)$ and $[\mathrm{SE}](t)$ normalized to $[\mathrm{SE}](0)$. It can be seen that $[\mathrm{PP}](0) /[\mathrm{SE}](0)$ strongly increases when tuning the pump from 650 to $840 \mathrm{~nm}$; indeed, it goes from 15 (Figure $2 \mathrm{~b}$ ) to $120 \%$, corresponding to an 
a)

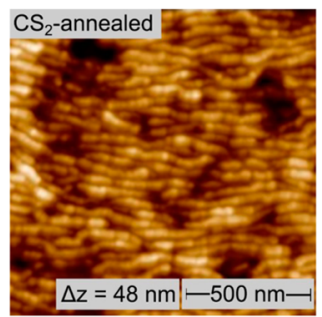

c)

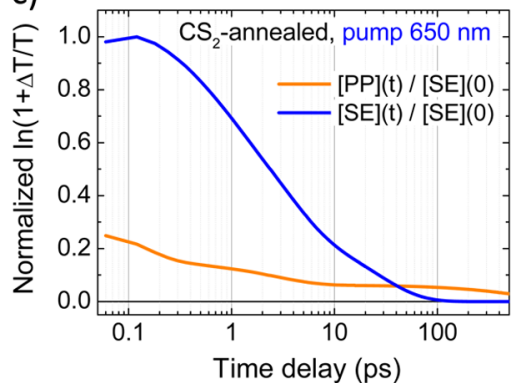

b)

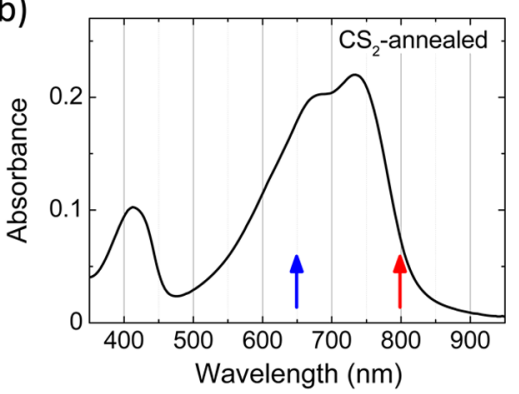

d)

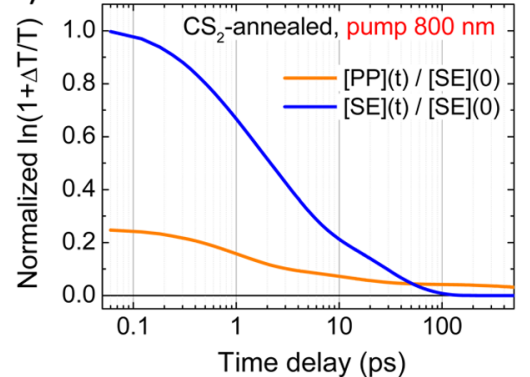

Figure 4. (a) AFM height image of sample- $\mathrm{CS}_{2}$-annealed. $\Delta z$ corresponds to the color scale. (b) Absorption spectrum of sample-CS ${ }_{2}$-annealed measured at room temperature $\left(25^{\circ} \mathrm{C}\right)$. The blue and red arrows indicate the different excitation wavelengths used in the pump-probe experiments. Panels $(\mathrm{c})$ and $(\mathrm{d})$ show the decay in time of $[\mathrm{PP}](t)$ and $[\mathrm{SE}](t)$ (see eqs 4 and 5 ) normalized to $[\mathrm{SE}](0)$, after excitation with a pulsed pump at 650 and $800 \mathrm{~nm}$, respectively. The corresponding $\Delta T / T$ spectra are shown in the Supporting Information (Figure S4).

8 -fold increase. Note that, not knowing the cross sections $\phi_{\mathrm{PP}}$ and $\phi_{\mathrm{SE}}$ (eq 6), we cannot deduce from our results that more PPs than SEs are photogenerated by the $840 \mathrm{~nm}$ pump. However, these results do show that the direct generation of PPs is substantially increased when aggregates are excited. The lifetimes of $[\mathrm{PP}](t)$ are shorter compared to that of the $650 \mathrm{~nm}$ excitation. Interestingly, no long-lived component is observed in this case. The stimulated emission signal in the $850-1050 \mathrm{~nm}$ wavelength range is significantly lower with respect to the $650 \mathrm{~nm}$ excitation, thus confirming that with $840 \mathrm{~nm}$ excitation, we obtain a significantly higher PPs to SEs ratio.

The same pump-probe experiments were performed on sample-CS $\mathrm{CS}_{2}$ which does not present a detectable amount of $\pi$ aggregates (Figure 1d). The results are summarized in Figure 3. In this case, no significant differences in the transient absorption spectra were observed upon tuning the laser pump wavelength from 650 to $800 \mathrm{~nm}$. For this sample, we had to use a shorter pump wavelength $(800 \mathrm{~nm})$ compared to sample-CB/DIO due to the optical absorption at $840 \mathrm{~nm}$ being too small. Figure $3 \mathrm{~b}, \mathrm{~d}$ shows the dynamics of PPs and SEs in the two experiments; only a slight increase in the value of $[\mathrm{PP}](0) /[\mathrm{SE}](0)$ is present, going from 20 to $30 \%$ when the pump at $800 \mathrm{~nm}$ is used. For both pump excitations, a long-lived component of $[\mathrm{PP}](t)$ is present, similarly to what was observed for sample-CB/DIO excited at $650 \mathrm{~nm}$ via the nonaggregated polymer chains. Contrary to sample-CB/DIO, which shows a decreased stimulated emission signal when passing from 650 to $840 \mathrm{~nm}$ pump, sample- $\mathrm{CS}_{2}$ shows a higher $\Delta T / T$ signal in the stimulated emission region $(850-1050 \mathrm{~nm}$ ) when the $800 \mathrm{~nm}$ pump is used (Figure 3c). We attribute part of this signal, especially the rise below $\sim 950 \mathrm{~nm}$, to scattered photons from the laser pump, which was not possible to completely remove, probably due to a slightly lower film quality compared to sample-CB/DIO. We estimate that, excluding the scattering contribution, the stimulated emission signal is roughly as intense as that with the $650 \mathrm{~nm}$ excitation (Figure 3a). This is expected, because the $[\mathrm{PP}](0) /[\mathrm{SE}](0)$ ratio shows only a marginal increase when passing to the $800 \mathrm{~nm}$ pump.

Finally, we investigated films of PCPDTBT that were postannealed in $\mathrm{CS}_{2}$ solvent vapor (hereafter called sample$\mathrm{CS}_{2}$-annealed). We have recently shown that spin-coated films that are postannealed in vapor of $\mathrm{CS}_{2}$ crystallize in a different crystal structure than films spin-coated from $\mathrm{CB} / \mathrm{DIO}{ }^{29}$ An AFM height image of sample- $\mathrm{CS}_{2}$-annealed is shown in Figure 4a. The film exhibits large-scale superstructures, composed of semicrystalline lamellae having uniform widths $(\sim 40 \mathrm{~nm})$ and uniform lengths $(0.5-1 \mu \mathrm{m})$. Our previous studies with TEM/ ED and GIWAXS suggested that no long-range $\pi$-aggregation is present in PCPDTBT films prepared as sample- $\mathrm{CS}_{2}$-annealed, even though the films look well-ordered and crystallized. ${ }^{29}$ Rather, a crystal structure with a strong interaction between pairs of polymer chains resulting in a dimer-like crystal structure was proposed, and a melting point of $280{ }^{\circ} \mathrm{C}$ was detected by differential scanning calorimetry (DSC) measurements. ${ }^{29}$ It has to be noted that no melting points were reported for PCPDTBT films before, and we also could not see any melting transitions for other spin-coated films (including those spin-coated using solvent additives such as DIO). Confirming the higher crystallinity, the absorption spectrum of sample-CS $\mathrm{CS}_{2}$-annealed shows bands with reduced width compared to the amorphous sample- $\mathrm{CS}_{2}$ (Figure $4 \mathrm{~b}$ ). However, the position of the bands resembles sample-CS $\mathrm{CS}_{2}$ and does not show the red-shifted band, which was instead observed in sample-CB/DIO and attributed to $\pi$-aggregates. ${ }^{28,31,32,35}$ This observation is in agreement with the absence of $\pi$-aggregates in this film and allows us to conclude that the intermolecular structures present in sample- $\mathrm{CS}_{2}$-annealed do not have a significant ground-state absorption.

Pump-probe experiments were performed on sample- $\mathrm{CS}_{2}$ annealed, exciting at 650 or $800 \mathrm{~nm}$. The evolution in time of $[\mathrm{PP}](t)$ and $[\mathrm{SE}](t)$ normalized to $[\mathrm{SE}](0)$ is shown in Figure 4c and $d$ for 650 and $800 \mathrm{~nm}$ excitation, respectively. The decays are very similar to those observed for sample- $\mathrm{CS}_{2}$, with the ratio $[\mathrm{PP}]$ 
(0)/[SE](0) being $25 \%$ when the $650 \mathrm{~nm}$ excitation is used. This ratio does not change when the excitation at $800 \mathrm{~nm}$ is used. In both samples, $[\mathrm{PP}](t)$ shows a long-lived component $(>400$ ps).

Our experiments on thin films of PCPDTBT show the impact of interchain $\pi$-aggregation on the process of direct photogeneration of PPs in neat polymer films. The ground-state optical absorption spectra reveal a considerable amount of $\pi$-aggregation in sample-CB/DIO, becoming negligible in sample- $\mathrm{CS}_{2}$ (Figure 1). The clear spectral separation of the absorption bands from $\pi$ aggregated and nonaggregated polymer chains allows their selective excitation in pump-probe experiments, using 650 and 800-840 nm laser pulses, respectively. In sample-CB/DIO, excitation of the $\pi$-aggregates $(840 \mathrm{~nm}$ ) results in a strong PIA signal from PPs at short delay times. In particular, we show in Figure $2 \mathrm{~d}$ that $[\mathrm{PP}](t)$ at time zero is as high as $120 \%$ of $[\mathrm{SE}](0)$. Strikingly, this percentage is 8 times higher than that in the case in which the nonaggregated chains are excited (650 nm pump, Figure $2 b$ ). These results clearly indicate that the excitation of $\pi$ aggregates results in much higher efficiency of direct PP generation, compared to the excitation of nonaggregated chains. In agreement with this observation, we show that the value of $[\mathrm{PP}](0) /[\mathrm{SE}](0)$ when the low photon energy excitation $(800$ $\mathrm{nm})$ is used decreases with a decreasing amount of $\pi$-aggregates in the films, going to $\sim 30 \%$ for sample- $\mathrm{CS}_{2}$ (Figure $3 \mathrm{~d}$ ) and $\sim 25 \%$ for sample- $\mathrm{CS}_{2}$-annealed (Figure $4 \mathrm{~d}$ ). When going from 650 to $800 \mathrm{~nm}$ excitation, sample- $\mathrm{CS}_{2}$ shows an increase in [PP] $(0) /[\mathrm{SE}](0)$, passing from $\sim 20$ to $\sim 30 \%$ (Figure $3 \mathrm{~b}, \mathrm{~d}$ ); this is much smaller than the 8 -fold increase observed for sample-CB/ DIO. We interpret this slight increase as originating from small amounts of $\pi$-aggregates in these films, not detectable in groundstate absorption spectroscopy. Finally, sample- $\mathrm{CS}_{2}$-annealed does not show any change in $[\mathrm{PP}](0) /[\mathrm{SE}](0)$ between the two excitation wavelengths, indicating the absence of $\pi$ aggregates in the film.

Note that for all samples, no difference in the rise time of the PIA signals of PPs and SEs was measured. This observation indicates that the photogeneration of the two species is prompt and occurs through independent channels, directly from the ground state within the $\sim 100$ fs time resolution of our setup. Only sample- $\mathrm{CS}_{2}$-annealed showed a slightly longer rise time (100 fs) of the SE signal when pumped at $650 \mathrm{~nm}$.

The long-lived tails ( $>400 \mathrm{ps}$ ) observed for all samples, excluding sample-CB/DIO when excited at $840 \mathrm{~nm}$, might be attributed to trapping of one or both charges constituting the PP, which would prevent them from quickly recombining. Additionally, a contribution from long-lived triplet states populated via intersystem crossing from $S_{1}$ or from PPs might be present in the same spectral region. Triplet states in this polymer present a PIA band strongly overlapped to that of PPs, ${ }^{37}$ which is therefore difficult to remove from the fitting of $\left(\Delta T / T_{\mathrm{PP}}\right)(\lambda, t)$.

We explain the observed influence of $\pi$-aggregation on direct PP generation as a result of the delocalization of the primary photoexcitation. The delocalization is facilitated by long-range $\pi$ aggregates, which have been shown to support interchain delocalized states. $^{42}$ Such states are more likely to have a polaronic character because the average distance between the electron and hole is increased compared to intrachain states. Additionally, $\pi$-aggregates present ground-state absorption (Figure 1). This last property is crucial and allows the direct generation via photon absorption from the ground state. We therefore propose the following scenario of prompt charge generation in neat polymer films: direct ground-state excitation of nonaggregated polymer chains results in the formation of SE and PP states on a time scale of $\sim 100 \mathrm{fs}$, with a strong prevalence of SE. When instead $\pi$-aggregated chains are excited, a strong increase in PP generation relative to SE is observed, thanks to the delocalized nature of the interchain states that are accessed. Further, we show that the presence of highly ordered semicrystalline lamellar regions not containing long-range $\pi$ stacking does not result in improved PP generation. This last observation further supports a picture where it is the direct photoexcitation of long-range $\pi$-aggregates that facilitates the generation of PPs. Indeed, in the case of aggregates that do not absorb light, as in sample- $\mathrm{CS}_{2}$-annealed, no effect of the longrange order on $\mathrm{PP}$ generation is observed.

In summary, by ultrafast pump-probe experiments, we have shown that the generation of PPs in neat conjugated polymer films is prompt ( $<100 \mathrm{fs})$ and that it is strongly favored when long-range $\pi$-aggregated chains are directly photoexcited, with the prompt PP to SE ratio showing an 8-fold increase compared to the case where nonaggregated chains are excited. We interpret this behavior in terms of the delocalized nature of the primary photoexcitation in $\pi$-aggregates. On the other hand, we show that if ground-state absorption from aggregates is absent, extended packing of chains to form larger highly ordered domains does not increase the efficiency of prompt PP pair generation. Our results indicate that interchain aggregates having ground-state absorption are the most important ingredient to promote the prompt generation of charges in neat polymer films. Finally, we stress that this mechanism might be of importance also in the photogeneration of charges in bulk heterojunctions for solar cells, which often contain large and relatively pure polymer phases.

\section{EXPERIMENTAL METHODS}

Materials. The PCPDTBT polymer was purchased from 1Material and used as received. The molecular weight was determined to be $M_{\mathrm{w}}=23 \mathrm{~kg} / \mathrm{mol}$ with a PDI of 1.7 (HT-SEC, $160{ }^{\circ} \mathrm{C}$, trichlorobenzene, against PS standards). All solvents $\left(\mathrm{CS}_{2}, \mathrm{CHCl}_{3}, \mathrm{CB}, \mathrm{DIO}\right)$ were purchased from Sigma-Aldrich (p.a. grade).

Sample Preparation. Films of (poly[2,6-(4,4-bis(2-ethylhexyl)4H-cyclopenta[2,1-b;3,4-b"] dithiophene)-alt-4,7-(2,1,3-benzothia diazole)]) (PCPDTBT) were spin-coated onto quartz substrates cleaned by $\mathrm{O}_{2}$ plasma $(10 \mathrm{~min}, 100 \mathrm{~W})$ from solutions of different solvents in a nitrogen atmosphere. The concentrations of the solutions were $3 \mathrm{mg} / \mathrm{mL}$. In the case of $\mathrm{CB}$, we added $2 \mathrm{wt} \%$ DIO to the solution. The final film thicknesses were in the range of $30-50 \mathrm{~nm}$. Sample- $\mathrm{CS}_{2}$-annealed was prepared on a quartz substrate by spin coating from a chloroform solution ( $3 \mathrm{mg} / \mathrm{mL}$ ) followed by $\mathrm{CS}_{2}$ solvent annealing at $90.5 \%$ solvent vapor pressure (as in ref 43 ).

Atomic Force Microscopy. AFM was performed on a Dimension Icon AFM from Bruker operating in tapping mode.

Absorption Spectroscopy. The absorbance of the films was measured using a Zeiss spectrometer (light source: CLH600; detector: MCS621 VIS II and MCS611 NIR 2,2 $\mu \mathrm{m}$ ) in transmission mode using fiber optics. The temperature was controlled by a hot-stage (THMS600, Linkam).

Ultrafast Spectroscopy. Time-resolved measurements were performed using a home-built femtosecond pump-probe spectrometer. During the measurements, samples were kept in a vacuum chamber $\left(10^{-4} \mathrm{mbar}\right)$ equipped with quartz windows. The system starts with a Ti:sapphire regenerative amplifier (Quantronix, Integra C), delivering $100 \mathrm{fs}$ pulses at a central wavelength of $800 \mathrm{~nm}$ with $1 \mathrm{~mJ}$ pulse energy at a repetition rate 
of $1 \mathrm{kHz}$. The overall time resolution of the setup can be estimated at $\sim 100$ fs. For the excitation pulses, a single-stage optical parametric amplifier (OPA), pumped at $400 \mathrm{~nm}$, allowed the choice of a desired pump wavelength from 500 to $900 \mathrm{~nm}$. Excitation pulses had a fluence of $\sim 5 \mu \mathrm{J} / \mathrm{cm}^{2}$; this low excitation fluence was chosen to provide a sufficient signal-to-noise ratio and at the same time minimize high excitation density effects. White light generated with a sapphire plate was used for probing in the NIR from 820 to $1500 \mathrm{~nm}$. The probe signal was detected by an optical multichannel analyzer working at the full $1 \mathrm{kHz}$ laser repetition rate.

\section{ASSOCIATED CONTENT}

\section{S Supporting Information}

UV-vis optical absorption spectra of PCPDTBT in solution and of thin films held at different temperatures, in situ spectroelectrochemistry, pump-probe spectra of sample- $\mathrm{CS}_{2}$-annealed, and tables summarizing the best-fit parameters of eqs 2 and 3. This material is available free of charge via the Internet at http://pubs. acs.org.

\section{AUTHOR INFORMATION}

\section{Corresponding Authors}

*E-mail: dinuzzo.daniele@tiscali.it (D.D.N.).

*E-mail: sabine.ludwigs@ipoc.uni-stuttgart.de (S.L.).

\section{Notes}

The authors declare no competing financial interest.

\section{ACKNOWLEDGMENTS}

D.D.N., F.F., S.L., and E.D.C. thank the DFG Priority Program SPP-1355 for funding. Discussions with M. Brinkmann on the crystal structure analysis are highly acknowledged. G.C. acknowledges support by the European Research Council Advanced Grant STRATUS (ERC-2011-AdG No. 291198). The research leading to these results has received funding from LASERLAB-EUROPE (Grant Agreement No. 284464, EC's Seventh Framework Programme).

\section{REFERENCES}

(1) Yu, G.; Gao, J.; Hummelen, J. C.; Wudl, F.; Heeger, A. J. Polymer Photovoltaic Cells - Enhanced Efficiencies via a Network of Internal Donor-Acceptor Heterojunctions. Science 1995, 270, 1789-1791.

(2) Barth, S.; Bassler, H. Intrinsic Photoconduction in PPV-Type Conjugated Polymers. Phys. Rev. Lett. 1997, 79, 4445-4448.

(3) Moses, D.; Dogariu, A.; Heeger, A. J. Ultrafast Detection of Charged Photocarriers in Conjugated Polymers. Phys. Rev. B 2000, 61, 9373-9379.

(4) Cerullo, G.; Lanzani, G.; De Silvestri, S.; Egelhaaf, H. J.; Luer, L.; Oelkrug, D. Primary Photoexcitations in Oligophenylenevinylene Thin Films Probed by Femtosecond Spectroscopy. Phys. Rev. B 2000, 62, 2429-2436.

(5) Dicker, G.; de Haas, M. P.; Siebbeles, L. D. A.; Warman, J. M. Electrodeless Time-Resolved Microwave Conductivity Study of ChargeCarrier Photogeneration in Regioregular Poly(3-hexylthiophene) Thin Films. Phys. Rev. B 2004, 70, 045203.

(6) Reid, O. G.; Malik, J. A. N.; Latini, G.; Dayal, S.; Kopidakis, N.; Silva, C.; Stingelin, N.; Rumbles, G. The Influence of Solid-State Microstructure on the Origin and Yield of Long-Lived Photogenerated Charge in Neat Semiconducting Polymers. J. Polym. Sci., Part B: Polym. Phys. 2012, 50, 27-37.

(7) Tautz, R.; Da Como, E.; Limmer, T.; Feldmann, J.; Egelhaaf, H.-J.; von Hauff, E.; Lemaur, V.; Beljonne, D.; Yilmaz, S.; Dumsch, I.; et al. Structural Correlations in the Generation of Polaron Pairs in LowBandgap Polymers for Photovoltaics. Nat. Commun. 2012, 3, 970.
(8) Reid, O. G.; Rumbles, G. Quantitative Transient Absorption Measurements of Polaron Yield and Absorption Coefficient in Neat Conjugated Polymers. J. Phys. Chem. Lett. 2013, 4, 2348-2355.

(9) Reid, O. G.; Pensack, R. D.; Song, Y.; Scholes, G. D.; Rumbles, G. Charge Photogeneration in Neat Conjugated Polymers. Chem. Mater. 2014, 26, 561-575.

(10) Magnanelli, T. J.; Bragg, A. E. Time-Resolved Raman Spectroscopy of Polaron Pair Formation in Poly(3-hexylthiophene) Aggregates. J. Phys. Chem. Lett. 2015, 6, 438-445.

(11) Tautz, R.; Da Como, E.; Wiebeler, C.; Soavi, G.; Dumsch, I.; Froehlich, N.; Grancini, G.; Allard, S.; Scherf, U.; Cerullo, G.; et al. Charge Photogeneration in Donor-Acceptor Conjugated Materials: Influence of Excess Excitation Energy and Chain Length. J. Am. Chem. Soc. 2013, 135, 4282-4290.

(12) Chen, W.; Xu, T.; He, F.; Wang, W.; Wang, C.; Strzalka, J.; Liu, Y.; Wen, J. G.; Miller, D. J.; Chen, J. H.; et al. Hierarchical Nanomorphologies Promote Exciton Dissociation in Polymer/Fullerene Bulk Heterojunction Solar Cells. Nano Lett. 2011, 11, 3707-3713.

(13) Maturova, K.; van Bavel, S. S.; Wienk, M. M.; Janssen, R. A. J.; Kemerink, M. Description of the Morphology Dependent Charge Transport and Performance of Polymer:Fullerene Bulk Heterojunction Solar Cells. Adv. Funct. Mater. 2011, 21, 261-269.

(14) Hedley, G. J.; Ward, A. J.; Alekseev, A.; Howells, C. T.; Martins, E. R.; Serrano, L. A.; Cooke, G.; Ruseckas, A.; Samuel, I. D. W. Determining the Optimum Morphology in High-Performance Polymer-Fullerene Organic Photovoltaic Cells. Nat. Commun. 2013, 4, 2867.

(15) Kouijzer, S.; Michels, J. J.; van den Berg, M.; Gevaerts, V. S.; Turbiez, M.; Wienk, M. M.; Janssen, R. A. J. Predicting Morphologies of Solution Processed Polymer:Fullerene Blends. J. Am. Chem. Soc. 2013, $135,12057-12067$

(16) Kroeze, J. E.; Savenije, T. J.; Vermeulen, M. J. W.; Warman, J. M. Contactless Determination of the Photoconductivity Action Spectrum, Exciton Diffusion Length, and Charge Separation Efficiency in Polythiophene-Sensitized $\mathrm{TiO}_{2}$ Bilayers. J. Phys. Chem. B 2003, 107, 7696-7705.

(17) Shaw, P. E.; Ruseckas, A.; Samuel, I. D. W. Exciton Diffusion Measurements in Poly(3-hexylthiophene). Adv. Mater. 2008, 20, 35163520.

(18) Kaake, L. G.; Jasieniak, J. J.; Bakus, R. C., II; Welch, G. C.; Moses, D.; Bazan, G. C.; Heeger, A. J. Photoinduced Charge Generation in a Molecular Bulk Heterojunction Material. J. Am. Chem. Soc. 2012, 134, 19828-19838.

(19) Falke, S. M.; Rozzi, C. A.; Brida, D.; Maiuri, M.; Amato, M.; Sommer, E.; De Sio, A.; Rubio, A.; Cerullo, G.; Molinari, E.; et al. Coherent Ultrafast Charge Transfer in an Organic Photovoltaic Blend. Science 2014, 344, 1001-1005.

(20) Jailaubekov, A. E.; Willard, A. P.; Tritsch, J. R.; Chan, W.-L.; Sai, N.; Gearba, R.; Kaake, L. G.; Williams, K. J.; Leung, K.; Rossky, P. J.; et al. Hot Charge-Transfer Excitons Set the Time Limit for Charge Separation at Donor/Acceptor Interfaces in Organic Photovoltaics. Nat. Mater. 2013, 12, 66-73.

(21) Bakulin, A. A.; Rao, A.; Pavelyev, V. G.; van Loosdrecht, P. H. M.; Pshenichnikov, M. S.; Niedzialek, D.; Cornil, J.; Beljonne, D.; Friend, R. H. The Role of Driving Energy and Delocalized States for Charge Separation in Organic Semiconductors. Science 2012, 335, 1340-1344.

(22) Sheng, C. X.; Tong, M.; Singh, S.; Vardeny, Z. V. Experimental Determination of the Charge/Neutral Branching Ratio $\eta$ in the Photoexcitation of $\pi$-Conjugated Polymers by Broadband Ultrafast Spectroscopy. Phys. Rev. B 2007, 75, 085206.

(23) Paquin, F.; Latini, G.; Sakowicz, M.; Karsenti, P.-L.; Wang, L.; Beljonne, D.; Stingelin, N.; Silva, C. Charge Separation in Semicrystalline Polymeric Semiconductors by Photoexcitation: Is the Mechanism Intrinsic or Extrinsic? Phys. Rev. Lett. 2011, 106, 197401.

(24) Shoaee, S.; Subramaniyan, S.; Xin, H.; Keiderling, C.; Tuladhar, P. S.; Jamieson, F.; Jenekhe, S. A.; Durrant, J. R. Charge Photogeneration for a Series of Thiazolo-Thiazole Donor Polymers Blended with the Fullerene Electron Acceptors PCBM and ICBA. Adv. Funct. Mater. 2013, 23, 3286-3298. 
(25) Martin, T. P.; Wise, A. J.; Busby, E.; Gao, J.; Roehling, J. D.; Ford, M. J.; Larsen, D. S.; Moule, A. J.; Grey, J. K. Packing Dependent Electronic Coupling in Single Poly(3-hexylthiophene) H- and JAggregate Nanofibers. J. Phys. Chem. B 2013, 117, 4478-4487.

(26) Sweetnam, S.; Graham, K. R.; Ndjawa, G. O. N.; Heumueller, T.; Bartelt, J. A.; Burke, T. M.; Li, W.; You, W.; Amassian, A.; McGehee, M. D. Characterization of the Polymer Energy Landscape in Polymer:Fullerene Bulk Heterojunctions with Pure and Mixed Phases. J. Am. Chem. Soc. 2014, 136, 14078-14088.

(27) Tremel, K.; Ludwigs, S. Morphology of P3HT in Thin Films in Relation to Optical and Electrical Properties. Adv. Polym. Sci. 2014, 265, $39-82$.

(28) Fischer, F. S. U.; Tremel, K.; Saur, A. K.; Link, S.; Kayunkid, N.; Brinkmann, M.; Herrero-Carvajal, D.; Lopez Navarrete, J. T.; Ruiz Delgado, M. C.; Ludwigs, S. Influence of Processing Solvents on Optical Properties and Morphology of a Semicrystalline Low Bandgap Polymer in the Neutral and Charged States. Macromolecules 2013, 46, 49244931.

(29) Fischer, F. S. U.; Trefz, D.; Back, J.; Kayunkid, N.; Tornow, B.; Albrecht, S.; Yager, K. G.; Singh, G.; Karim, A.; Neher, D.; et al. Highly Crystalline Films of PCPDTBT with Branched Side Chains by Solvent Vapor Crystallization: Influence on Opto-Electronic Properties. Adv. Mater. 2015, 27, 1223.

(30) van Stokkum, I. H. M.; Larsen, D. S.; van Grondelle, R. Global and Target Analysis of Time-Resolved Spectra. Biochim. Biophys. Acta 2004, 1657, 82-104.

(31) Peet, J.; Cho, N. S.; Lee, S. K.; Bazan, G. C. Transition from Solution to the Solid State in Polymer Solar Cells Cast from Mixed Solvents. Macromolecules 2008, 41, 8655-8659.

(32) Gu, Y.; Wang, C.; Russell, T. P. Multi-Length-Scale Morphologies in PCPDTBT/PCBM Bulk-Heterojunction Solar Cells. Adv. Energy Mater. 2012, 2, 683-690.

(33) Rogers, J. T.; Schmidt, K.; Toney, M. F.; Kramer, E. J.; Bazan, G. C. Structural Order in Bulk Heterojunction Films Prepared with Solvent Additives. Adv. Mater. 2011, 23, 2284-2288.

(34) Morana, M.; Azimi, H.; Dennler, G.; Egelhaaf, H.-J.; Scharber, M.; Forberich, K.; Hauch, J.; Gaudiana, R.; Waller, D.; Zhu, Z.; et al. Nanomorphology and Charge Generation in Bulk Heterojunctions Based on Low-Bandgap Dithiophene Polymers with Different Bridging Atoms. Adv. Funct. Mater. 2010, 20, 1180-1188.

(35) Agostinelli, T.; Ferenczi, T. A. M.; Pires, E.; Foster, S.; Maurano, A.; Mueller, C.; Ballantyne, A.; Hampton, M.; Lilliu, S.; Campoy-Quiles, M.; et al. The Role of Alkane Dithiols in Controlling Polymer Crystallization in Small Band Gap Polymer:Fullerene Solar Cells. J. Polym. Sci., Part B: Polym. Phys. 2011, 49, 717-724.

(36) Hwang, I.-W.; Soci, C.; Moses, D.; Zhu, Z.; Waller, D.; Gaudiana, R.; Brabec, C. J.; Heeger, A. J. Ultrafast Electron Transfer and Decay Dynamics in a Small Band Gap Bulk Heterojunction Material. Adv. Mater. 2007, 19, 2307-2312.

(37) Di Nuzzo, D.; Aguirre, A.; Shahid, M.; Gevaerts, V. S.; Meskers, S. C. J.; Janssen, R. A. J. Improved Film Morphology Reduces Charge Carrier Recombination into the Triplet Excited State in a Small Bandgap Polymer-Fullerene Photovoltaic Cell. Adv. Mater. 2010, 22, 43214324.

(38) Etzold, F.; Howard, I. A.; Forler, N.; Cho, D. M.; Meister, M.; Mangold, H.; Shu, J.; Hansen, M. R.; Muellen, K.; Laquai, F. The Effect of Solvent Additives on Morphology and Excited-State Dynamics in PCPDTBT:PCBM Photovoltaic Blends. J. Am. Chem. Soc. 2012, 134, $10569-10583$.

(39) Nikitenko, V. R.; Hertel, D.; Bässler, H. Dispersive Geminate Recombination in a Conjugated Polymer. Chem. Phys. Lett. 2001, 348, $89-94$.

(40) Offermans, T.; Meskers, S. C. J.; Janssen, R. A. J. Charge Recombination in a Poly(para-phenylene vinylene)-Fullerene Derivative Composite Film Studied by Transient, Nonresonant, Hole-Burning Spectroscopy. J. Chem. Phys. 2003, 119, 10924-10929.

(41) Albert-Seifried, S.; Ko, D.-H.; Huettner, S.; Kanimozhi, C.; Patil, S.; Friend, R. H. Efficiency Limitations in a Low Band-Gap
Diketopyrrolopyrrole-Based Polymer Solar Cell. Phys. Chem. Chem. Phys. 2014, 16, 6743-6752.

(42) Osterbacka, R; An, C. P.; Jiang, X. M.; Vardeny, Z. V. TwoDimensional Electronic Excitations in Self-Assembled Conjugated Polymer Nanocrystals. Science 2000, 287, 839-842.

(43) Faist, M. A.; Kirchartz, T.; Gong, W.; Ashraf, R. S.; McCulloch, I.; de Mello, J. C.; Ekins-Daukes, N. J.; Bradley, D. D. C.; Nelson, J. Competition between the Charge Transfer State and the Singlet States of Donor or Acceptor Limiting the Efficiency in Polymer:Fullerene Solar Cells. J. Am. Chem. Soc. 2012, 134, 685-692. 